\title{
Review of dark photon searches
}

\author{
Achim Denig ${ }^{1, \star}$ \\ ${ }^{1}$ Institute for Nuclear Physcis and PRISMA Cluster of Excellence, \\ Johannes Gutenberg University Mainz, Germany
}

\begin{abstract}
Dark Photons are hypothetical extra-U(1) gauge bosons, which are motivated by a number of astrophysical anomalies as well as the presently seen deviation between the Standard Model prediction and the direct measurement of the anomalous magnetic moment of the muon, $(g-2)_{\mu}$. The Dark Photon does not serve as the Dark Matter particle itself, but acts as a messenger particle of a hypothetical Dark Sector with residual interaction to the Standard Model. We review recent Dark Photon searches, which were carried out in a global effort at various hadron and particle physics facilities. We also comment on the perspectives for future invisble searches, which directly probe the existence of Light Dark Matter particles.
\end{abstract}

\section{Motivation}

Dark Photons are hypothetical particles of an extra U(1) gauge group. Such extra U(1) gauge groups are predicted by almost any extension of the Standard Model. Indeed, the related extra U(1) gauge bosons are searched for from the lowest (e.g. searches for axion-like particles) up to the highest energies at the LHC. Recently, the mass range for a vector particle in the $\mathrm{MeV}$ to $\mathrm{GeV}$ scale has been in the focus of interest due to the observation by Arkani Hamed et al. [1] that particles of such a mass scale might explain a surprisingly large number of astrophysical anomalies. In addition, it was realized that a Dark Photon of such a mass might explain the presently seen deviation between the direct measurement and the Standard Model prediction of the anomalous magnetic moment of the muon, $(g-2)_{\mu}[2]$.

Fig. 1 shows two Feynman diagrams with Dark Photon interactions. The upper diagram shows the kinetic mixing between the Standard Model photon and the hypothetical Dark Photon $\gamma^{\prime}$ (aka $A^{\prime}$, $Z^{\prime}$, or U boson) via a loop of charged leptons, $L$. Such a kinetic mixing term was propsed already in the 80's by Holdom [3]. The lower diagram might give rise to the positron excess presently seen in the spectrum of cosmic rays, which is indeed one of the most striking astrophysical anomalies. Dark Matter (DM) particles annihilate via a Dark Photon into an $e^{+} e^{-}$pair. Within the kinetic mixing model two free parameters remain: the mass of the Dark Photon, $m_{\gamma^{\prime}}$, and the mixing parameter $\epsilon$, which parametrizes the strength of the coupling of the Dark Photon to ordinary Standard Model matter: $\epsilon^{2}=\alpha^{\prime} / \alpha$, where $\alpha$ is the QED fine structure constant.

The possible relation of the Dark Photon to the Dark Matter problem as well as the fact that it might explain the $(g-2)_{\mu}$ puzzle, triggered an enormous theoretical and experimental interest in the particle

^e-mail: denig@kph.uni-mainz.de 
and hadron physics community. In the following we will discuss electron scattering experiments as well as results from hadron and $e^{+} e^{-}$accelerators. Very recently, an anomaly was observed in a nuclear decay of Berryllium [4]. This could be a first hint for a $17 \mathrm{MeV}$ Dark Photon signal, however the confirmation of the anomaly by an independent group is pending.

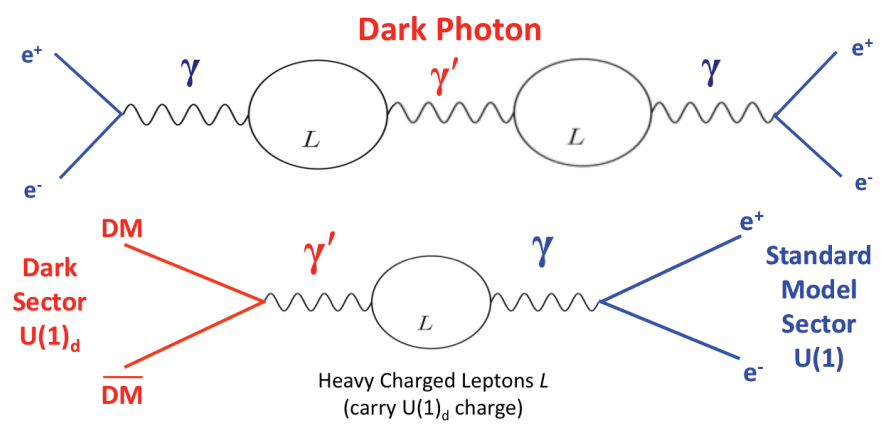

Figure 1. Feynman graphs depicting interactions via a hypothetical Dark Photon $\gamma^{\prime}$. Up: Kinetic mixing model; Down: Interaction between the Standard Model sector and the Dark Sector via a Dark Photon. The loop denotes a pair of charged leptons, which couple both to the Standard Model photon as well as the Dark Photon.

\section{Searches using fixed-target experiments}

As Bjoerken and collaborators [5] have pointed out, low-energy electron accelerators in combination with high-resolution detectors are very well suited for Dark Photon searches. By scattering the electron beam on a nuclear target, the Dark Photon may be emitted in the initial or final state, see Fig. 2. Its coupling to an $e^{+} e^{-}$pair allows for an identification by looking for a bump in the $e^{+} e^{-}$ invariant mass. The huge background is almost entirely given by QED contributions, such as for instance Bethe-Heitler processes. In order to achieve competitive results in these kind of experiments, the following conditions need to be fulfiled:

- High intensity electron beams (in the order of hundreds of microamps) are needed.

- An excellent mass resolution is needed as obtained for instance with high resolution magnetic spectrometers.

- The kinematics of the signal process requires a detection of electrons and positrons in the final state at small angles.

Successful pilot experiments have been carried out in 2011 at MAMI in Mainz [6] (A1 experiment) and JLAB [7] (APEX experiment) with electron beam energies of $0.9 \mathrm{GeV}$ and $2.3 \mathrm{GeV}$, respectively. These runs could improve upon existing Dark Photon limits from BaBar (2009 results) in the mass range around $200 \mathrm{MeV}$. More recently, a very wide parameter range between approximately $40 \mathrm{MeV}$ and $200 \mathrm{MeV}$ was tested at MAMI with the high resolution spectrometer (HRS) setup A1 [8]. No significant signal was found and the $\epsilon$ parameter range down to $10^{-3}$ was excluded, constraining a large part of the parameter range motivated by $(g-2)_{\mu}$, see Fig. 4.

For the near and mid-term future several dedicated experiments are in preparation at JLAB. The APEX experiment [9], which is using an existing HRS setup at JLAB, will extend the mass range covered by A1/MAMI towards higher masses and lower $\epsilon$ values. The HPS experiment [10] will exploit a 

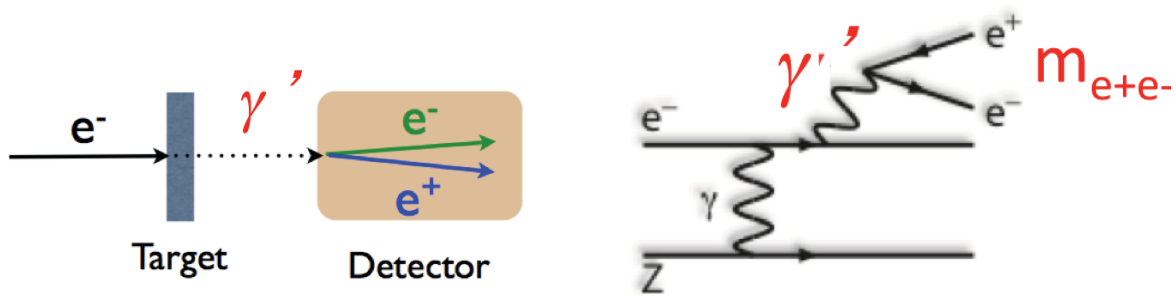

Figure 2. Poduction of a Dark Photon in a fixed-target environment. Left: Sketch of the experiment. Right: Feynman diagram for the production of the Dark Photon. One important background process is given by exchanging the Dark Photon with a QED photon.

displaced vertex technique, which allows to test even lower values of $\epsilon$. First data has been collected with the HPS setup in 2016. Finally, the Dark Light [11] experiment at the FEL accelerator at JLAB aims for testing the low mass region below the results already covered by A1. The new spectrometer setup MAGIX at the MESA accelerator [12] in Mainz will also be able to cover this mass range. Both MAGIX and Dark Light are planned to operate at modern Energy-Recovering-Linac accelerators which provide the highest intensities. High resolution measurements can be expected due to the fact that windowless gas targets will be used, which minimize effects of multiple scattering.

\section{Data mining campaigns using existing data sets}

The recent interest in $\mathrm{MeV}$ to $\mathrm{GeV}$ scale Dark Photons triggered also a number of searches at electronpositron as well as hadron accelerators using already existing data sets, which were collected for completely different purposes (data mining campaigns). Essentially, any process in which within the Standard Model a (virtual) photon couples to a pair of electrons, muons, or hadrons, can be used as a probe to search for Dark Photons. The following investigations came out to be most successful in producing competitive exclusion limits:

- Radiative decays of vector resonances: The BABAR collaboration had first explored this technique by looking for bumps in the invariant mass spectrum of muon pairs in radiative decays of the $\Upsilon(3 S)$ resonance [13]. Later, the KLOE collaboration investigated decays of the $\phi$ resonance into an $\eta$ meson and a pair of $e^{+} e^{-}[14]$.

- Dalitz decays of the $\pi^{0}$ and $\eta$ mesons: This allows to explore the low mass range, see also Fig. 3 (left). The method was applied by the NA48 collaboration at the SPS/CERN [15], by WASA at COSY [16], HADES at GSI [17], as well as PHENIX at RHIC [18].

- Initial State Radiation (ISR): A wide range of Dark Photon masses can be probed by looking for Dark Photons, which are produced via the ISR process, see Fig. 3 (right). KLOE has searched for the Dark Photon below $1 \mathrm{GeV}$ in the $\mu^{+} \mu^{-}$[19], the $e^{+} e^{-}$[20], and more recently also in the $\pi^{+} \pi^{-}$ final states [21]. A particulrily competitive parameter range was probed by BABAR below $10 \mathrm{GeV}$ by searching for peaks in the radiative $\mu^{+} \mu^{-}$and $e^{+} e^{-}$spectrum [22].

A compilation of all exclusion plots obtained in the last years can be found in Fig. 4. Constraints from old axion searches (experiments E141 and E774) as well as a constraint from the precision observables (g-2) of the electron, are also shown. The red band depicts the parameter range, in which a Dark Photon could explain the presently seen deviation of 3.6 standard deviations between the 

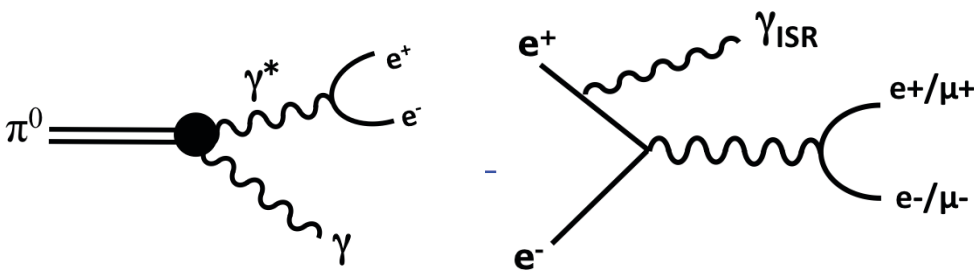

Figure 3. A search for the Dark Photon can be carried out in any process, in which a virtual photon couples to a lepton or quark pair. By exchanging the QED photon with a Dark Photon, one can look for a bump in the invariant mass spectrum of the lepton/quark pair. Left: $\pi^{0}$ Dalitz decay; Right: ISR production of the Dark Photon.

Standard Model prediction and the direct measurement of the $(g-2)$ of the muon. In the meantime, the $(g-2)_{\mu}$ band is completely covered by experiments.

For the future, the BELLE II project will collect a statistics, which will exceed the BABAR data set by almost two orders of magnitude, which will further constrain the Dark Photon parameter range towards lower $\epsilon$ values. At low Dark Photon masses, it can be expected that Dalitz decay studies at $\mathrm{LHCb}$ or elsewhere as well as the already mentioned low-energy fixed-target experiments will yield competitive results. The HPS project is designed to resolve displaced vertices, which will test islands of very low $\epsilon$ at masses below $500 \mathrm{MeV}$. A recent summary of the already published searches as well as the future experiments can be found in Ref. [23].

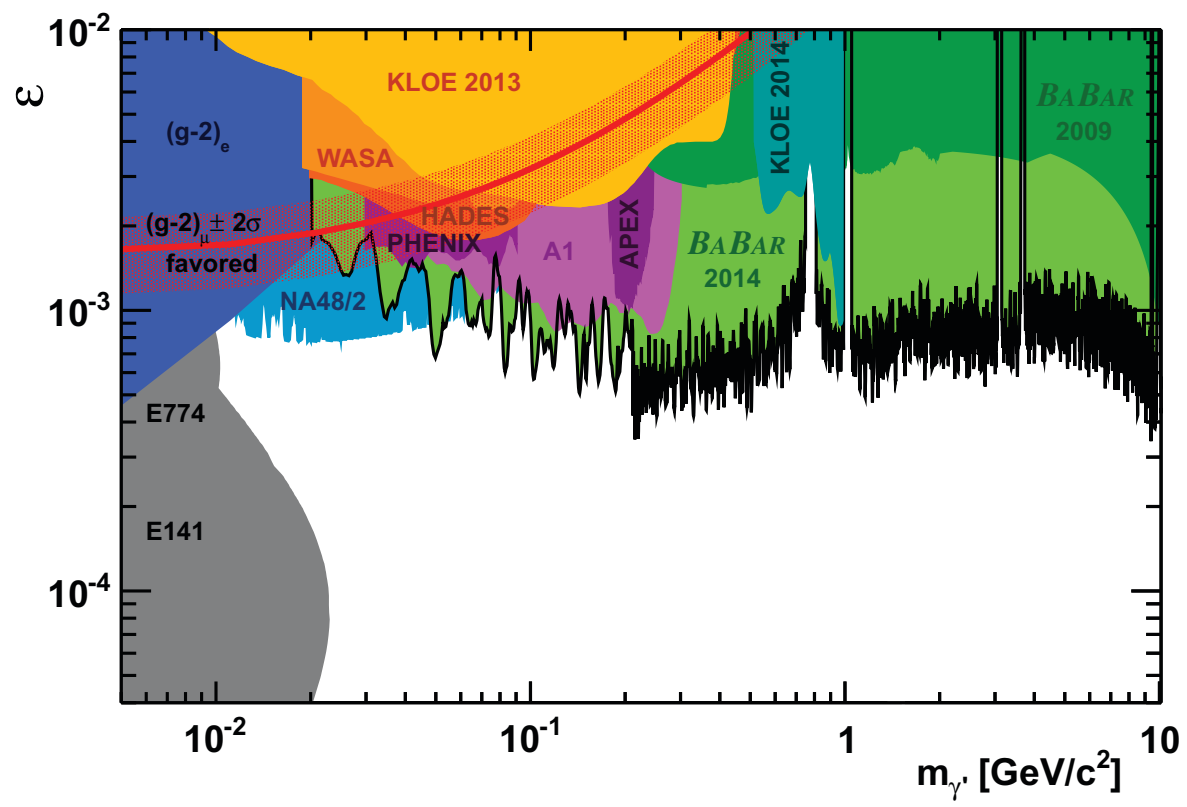

Figure 4. Compilation of existing Dark Photon constraints in the parameter range Dark Photon coupling, $\epsilon$, versus the mass of the Dark Photon, $m_{\gamma^{\prime}}$. 


\section{Invisible dark photon decays}

The kinetic mixing model discussed so far, predicts a Dark Photon, which kinematically mixes with the ordinary Standard Model photon. As mentioned in the beginning, such a Dark Photon not necessarily needs to be related to the Dark Matter problem, but astrophysical observations motivate the existence of a GeV scale Dark Photon. In the kinematic mixing model, the Dark Photon has a mass, which is small compared to the Dark Matter candidate. A feature of the kinematic mixing model is that the Dark Photon decays to Standard Model particles (leptons and quarks), however suppressed by a factor of $\epsilon$ in comparison to the ordinary photon coupling.

We would like to discuss also a second model, in which the Dark Photon is actually heavier than the Dark Matter particle. In this case the Dark Photon can decay to the Dark Matter candidate directly and hence does not primarily decay to Standard Model particles. Fig. 5 shows the production and decay of such a Dark Photon in a fixed target scattering experiment. Due to the fact that the Dark Photon decays invisibly in the experiment, we call this model the Invisible Dark Photon model. It should be stressed that theoretically Light (i.e. MeV scale) Dark Matter is a vital option for the Dark Matter problem. Present direct Dark Matter searches are however not sensitive to such light particles.

A Dark Photon in the Invisible Dark Photon model is experimentally much less constraint than a Dark Photon in the kinematic mixing model. This calls for new experiments and in fact quite a number of them are actually prepared at various laboratories worldwide (Cornell, JLAB, Mainz, Frascati, CERN, Novosibirsk) [23]. A common feature of these projects is the search for large missing mass and/or missing momentum in scattering events. Again, several experimental environments can be exploited, ranging from electron-positron annihilation to fixed target experiments.

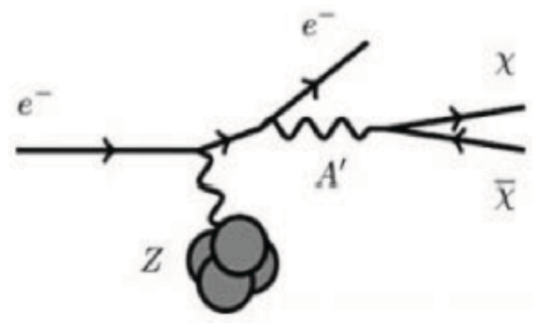

Figure 5. Invisible Dark Photon decay into a pair of Dark Matter candidates, $\chi \bar{\chi}$. Differently from previous plots the Dark Photon is shere denoted as $A^{\prime}$.

An exciting idea is to search for the Dark Matter candidates directly in a fixed target experiments as depicted in Fig. 5. This might be achieved very effectively by carrying out these searches behind the beam dumps of high intensity electron accelerators, so-called Beam Dump Experiments (BDX) Ref. [24]. High-intensity accelerators, as the ones operated at JLAB or Mainz, have the potential to produce per year in the order of $10^{21}$ electrons on target (EOT). For such kind of searches the experimental techniques to detect the LDM candidate could be similar to the techniques used in classical Dark Matter experiments. It yet remains to be seen whether the beam-related background (e.g. neutrons) is low enough to successfully carry out the experiments.

\section{Summary and outlook}

Searches for Dark Photons, which are hypothetical vector particles of an extra U(1) gauge sector, are motivated by the fact that they might explain a number of astrophysical anomalies, such as for 
instance the excess of positrons in cosmic rays. The Dark Photon is not a candidate for the Dark Matter particle itself, but serves as a gauge particle of a hypothetical Dark Sector. In the last years, we have witnessed a huge experimental activity worldwide to search for such a Dark Photon. Presently, the only evidence stems from a nuclear transition in Beryllium, in which an evidence for a $17 \mathrm{MeV}$ particle is claimed. it will be most exciting to see whether this evidence is confirmed by yet another group.

Recent Dark Photon searches were also motivated by the fact that a $\mathrm{MeV}$ to $\mathrm{GeV}$ scale Dark Photon could serve as an explanation for the deviation presently seen between the Standard Model prediction and the direct measurement of $(g-2)_{\mu}$. In the meantime, such a possibility is ruled out in the kinetic mixing model, in which the Dark Photon couples primarily to lepton pairs (or depending on its mass also to quark pairs).

A new generation of experiments is in preparation to explore Dark Photon searches, in which the Dark Photon decays primarily to Dark Matter itself. Such kind of experiments open the avenue to search for Light Dark Matter, which so far is not constraint by classical Dark Matter experiments. In this Invisible Dark Photon model an explanation for the $(g-2)_{\mu}$ discrepancy is still given. A recent summary report describes in detail the future directions in the field of Dark Photon searches and contains a full list of references of Dark Photon experiments and of theoretical papers related to the subject. We invite the interested reader to have a look at this summary report Ref. [23].

\section{References}

[1] N. Arkani-Hamed, D. P. Finkbeiner, T. R. Slatyer and N. Weiner, Phys. Rev. D 79015014 (2009)

[2] M. Pospelov, Phys. Rev. D 80095002 (2009)

[3] B. Holdom, Phys. Lett. B 166196 (1986)

[4] A. J. Krasznahorkay et al., Phys. Rev. Lett. 116042501 (2016)

[5] J. D. Bjorken, R. Essig, P. Schuster and N. Toro, Phys. Rev. D 80075018 (2009)

[6] H. Merkel et al. [A1 Collaboration], Phys. Rev. Lett. 106251802 (2011)

[7] S. Abrahamyan et al. [APEX Collaboration], Phys. Rev. Lett. 107191804 (2011)

[8] H. Merkel et al. [A1 Collaboration], Phys. Rev. Lett. 106251802 (2011)

[9] R. Essig, P. Schuster, N. Toro and B. Wojtsekhowski, JHEP 1102009 (2011)

[10] http://nuclear.unh.edu/HPS/HPS_proposal.pdf.

[11] http://dmtpc.mit.edu/DarkLight/DarkLightProposal_PAC39.pdf.

[12] K. Aulenbacher, M. Dehn, H. J. Kreidel, R. Heine and R. Eichhorn, ICFA Beam Dyn. Newslett. 58145 (2012)

[13] B. Aubert et al. [BaBar Collaboration], Phys. Rev. Lett. 103081803 (2009)

[14] D. Babusci et al. [KLOE-2 Collaboration], Phys. Lett. B 720111 (2013)

[15] J. R. Batley et al. [NA48/2 Collaboration], Phys. Lett. B 746178 (2015)

[16] P. Adlarson et al. [WASA-at-COSY Collaboration], Phys. Lett. B 726187 (2013)

[17] G. Agakishiev et al. [HADES Collaboration], Phys. Lett. B 731265 (2014)

[18] A. Adare et al. [PHENIX Collaboration], Phys. Rev. C 91031901 (2015)

[19] D. Babusci et al. [KLOE-2 Collaboration], Phys. Lett. B 736459 (2014)

[20] A. Anastasi et al., Phys. Lett. B 750633 (2015)

[21] A. Anastasi et al. [KLOE-2 Collaboration], Phys. Lett. B 757356 (2016)

[22] J. P. Lees et al. [BaBar Collaboration], Phys. Rev. Lett. 113201801 (2014)

[23] J. Alexander et al., arXiv:1608.08632 [hep-ph]

[24] E. Izaguirre, G. Krnjaic, P. Schuster and N. Toro, Phys. Rev. D 90014052 (2014) 